\title{
Influência do Tempo de Internamento sobre a Força Muscular Respiratória e Nível Funcional de Adultos com Leucemia e Linfoma
}

https://doi.org/10.32635/2176-9745.RBC.2018v64n4.202

Influence of Hospitalization Time about Respiratory Muscle Strength and Functional Level of Adults with Leukemia and Lymphoma

Influencia del Tiempo de Internamiento sobre la Fuerza Muscular Respiratoria y Nivel Funcional de Adultos con Leucemia y Linfoma

Natali dos Santos Nascimento'; Nicolly Del Carmen Parra Molina Mattos²; Samara de Souza Marques ${ }^{3}$; Thais Conceição Cruz ${ }^{4}$; Camila Reinbold Rezende ${ }^{5}$; Cássio Magalhães da Silva e Silva ${ }^{6}$

Resumo

Introduçáo: As neoplasias hematológicas, leucemias e linfomas são patologias que afetam o sangue ou tecidos formadores dele. Durante o período de hospitalização, os pacientes podem desenvolver redução da capacidade funcional que pode interferir na sua função respiratória. Objetivo: Avaliar a influência do tempo de internamento sobre a força muscular respiratória e o nível funcional de adultos com leucemia e linfoma. Método: Estudo observacional, com delineamento longitudinal e abordagem quantitativa, realizado na enfermaria onco-hematológica do Complexo Hospitalar Universitário Professor Edgard Santos (Hupes). A avaliação da força muscular respiratória foi mensurada pelo manovacuômetro e a capacidade funcional pela escala de desempenho de Karnofsky (KPS). Resultados: No decorrer do tempo de internamento dos pacientes, houve uma diminuição da pressão expiratória máxima $(\mathrm{PEM})(\mathrm{p}=0,000)$, porém não foi observada diferença significativa na pressão inspiratória máxima (PIM) ( $>0,05)$. Em relação à KPS, os pacientes apresentaram nível de funcionalidade de 70\%. Conclusão: Este estudo demonstrou que a PEM foi alterada durante o internamento, porém não houve modificação da PIM e da funcionalidade dos pacientes.

Palavras-chave: Leucemia; Linfoma; Avaliação de Estado de Karnofsky; Tempo de Internaçáo; Força Muscular.

\section{Abstract}

Introduction: Hematologic neoplasms, leukemias and lymphomas are pathologies that affect the blood or tissues that form it. During the hospitalization period patients may develop functional capacity reduction, which may interfere with their respiratory function. Objective: Evaluate the influence of hospitalization time about respiratory muscle strength and functional level of adults with leukemia and lymphoma. Method: Observational study, with longitudinal design and quantitative approach, performed at the onco-hematological ward of the University Hospital Complex Professor Edgard Santos (Hupes). The assessment of respiratory muscle strength was measured using the manovacuometer and functional capacity using the Karnofsky Performance Scale (KPS). Results: During the hospitalization time, there was a decrease in the maximum expiratory pressure (PEM) $(\mathrm{p}=0.000)$, but no significant difference was observed in the maximum inspiratory pressure (PIM) $(\mathrm{p}>0.05)$. In relation to KPS, the patients presented functional level of $70 \%$. Conclusion: This study demonstrated that PEM was altered during hospitalization, but there was no modification of the PIM and the functionality of the patients. Key words: Leukemia; Lymphoma; Karnofsky Performance Status; Length of Stay; Muscle Strength.

\section{Resumen}

Introducción: Las neoplasias hematológicas, leucemias y linfomas son patologías que afectan a la sangre o tejidos formadores de él. Durante el período de hospitalización los pacientes pueden desarrollar una reducción de la capacidad functional, que puede interferer en su función respiratoria. Objetivo: Evaluar la influencia del tiempo de internamiento sobre la fuerza muscular respiratoria y nivel funcional de adultos con leucemia y linfoma. Método: Estudio observacional, con delineamiento longitudinal y el enfoque cuantitativo, realizado en la enfermería onco-hematológica del Complejo Hospitalario Universitario Profesor Edgard Santos (Hupes). La evaluación de la fuerza muscular respiratoria se midió utilizando el manovacuómetro y la capacidad funcional utilizando la escala de rendimiento de Karnofsky (KPS). Resultados: En el transcurso del tiempo de internamiento de los pacientes, hubo una disminución de la presión espiratoria máxima (PEM) $(\mathrm{p}=0,000)$, pero no se observó diferencia significativa en la presión inspiratoria máxima (PIM) $(\mathrm{p}>0,05)$. En relación a KPS, los pacientes presentaron un nivel de funcionalidad del 70\%. Conclusión: Este estudio demostró que la PEM fue alterada durante el internamiento, pero no hubo modificación de la PIM y de la funcionalidad de los pacientes.

Palabras clave: Leucemia; Linfoma; Estado de Ejecución de Karnofsky; Tiempo de Internación; Fuerza Muscular.

\footnotetext{
${ }^{1}$ Universidade Federal da Bahia (UFBA). Salvador (BA), Brasil. Orcid iD: https://orcid.org/0000-0002-3473-4921

2 UFBA. Salvador (BA), Brasil. Orcid iD: https://orcid.org/0000-0002-8533973

${ }^{3}$ UFBA. Salvador (BA), Brasil. Orcid iD: https://orcid.org/0000-0002-103707907

${ }^{4}$ UFBA. Salvador (BA), Brasil. Orcid iD: https://orcid.org/0000-0002-47002885

${ }^{5}$ Hospital Universitário Professor Edgar Santos (Hupes). Salvador (BA), Brasil. Orcid iD: https://orcid.org/0000-0003-1989-2531

${ }^{6}$ UFBA. Salvador (BA), Brasil. Orcid iD: https://orcid.org/0000-0002-9119-5418

Endereço para correspondência: Natali dos Santos Nascimento. Departamento de Fisioterapia da UFBA. Instituto de Ciências da Saúde. Av. Reitor Miguel Calmon s/n - Vale do Canela. Salvador (BA), Brasil. CEP 40110-100. E-mail: natali.nascimento16@gmail.com
} 


\section{INTRODUÇÃO}

As neoplasias hematológicas, leucemias e linfomas são patologias que afetam o sangue ou seus tecidos formadores ${ }^{1}$. A estimativa de novos casos de leucemias é de 5.940 novos casos em homens e 4.860 em mulheres, além de 5.370 casos novos de linfoma não Hodking em homens e 4.810 em mulheres; já os casos novos de linfoma de Hodking correspondem a $1.480 \mathrm{em}$ homens e 1.050 em mulheres para cada ano do biênio 2018-2019².

Nesse sentido, tanto o adoecimento quanto o tratamento quimioterápico são responsáveis por alterar o metabolismo dos pacientes com neoplasia, sendo comum a redução da nutrição com consequente diminuição da massa muscular, fator responsável por fraqueza difusa ${ }^{3,4}$. Além disso, a força muscular respiratória pode ser modificada, com redução do movimento do músculo diafragma e expansibilidade torácica, o que induz a alteração do mecanismo da tosse $e^{5,6}$.

A avaliação da força muscular respiratória é um teste simples que possibilita mensurar a força e realizar detecção precoce da presença de debilidade muscular, visto que a fraqueza é uma situaçáo que, a depender do grau, culmina em redução da ventilação pulmonar, capacidade vital, e favorece a morbidade por infeçóes 7 . O uso de escalas específicas constitui uma conduta com vantagem de segurança e baixo custo, que são amplamente utilizadas na prática clínica para avaliação da funcionalidade ${ }^{8}$.

O nível funcional é um preditor de saúde que determina as habilidades físicas do indivíduo, possibilitando, dessa maneira, um bem-estar, pois consiste na competência do gerenciamento da vida, em aspectos que englobam habilidades para realizar as atividades básicas de vida diária (ABVD), como banho, vestimenta, autocuidado e mobilidade, até atividades instrumentais de vida diária (AIVD) com aptidão para fazer e resolver questóes com enfoque na participação direta do indivíduo em sociedade, ligado ao grau de independência ${ }^{9,10}$. A escala de desempenho de Karnofsky (KPS) em oncologia é empregada na tomada de decisóes e prognóstico, permitindo classificar os pacientes de acordo seu nível funcional ${ }^{8}$.

Durante o período de hospitalização, os pacientes podem desenvolver reduçáo da capacidade funcional, que pode interferir na sua função respiratória, aumentando os índices de morbimortalidade ${ }^{11}$. As publicaçóes sobre avaliação da força muscular respiratória, capacidade e comprometimento funcional de pacientes oncológicos são escassas; por isso, a possibilidade de avaliá-los ao longo do tempo amplia o conhecimento na disponibilização de informaçóes relevantes de pesquisa em saúde, permitindo entender sua evolução durante a permanência hospitalar.
Além disso, corrobora os subsídios para a assistência e o tratamento, viabilizando, assim, a tomada de decisão em propostas terapêuticas baseadas em evidências.

O objetivo deste estudo foi avaliar a influência do tempo de internamento sobre a força muscular respiratória e o nível funcional de adultos com leucemia e linfoma.

\section{MÉTODO}

Trata-se de um estudo observacional, com delineamento longitudinal e abordagem quantitativa, realizado em uma unidade hospitalar, de alta complexidade, do Complexo Hospitalar Universitário Professor Edgard Santos (Hupes), no período de fevereiro a julho de 2017. Este estudo faz parte do projeto de pesquisa aprovado pelo Comitê de Ética em Pesquisa do Hupes da Universidade Federal da Bahia sob o parecer número 1.805.649. O estudo respeitou os aspectos Éticos de Pesquisa envolvendo seres humanos em concordância com a resolução CNS 466/12. Os pacientes receberam informaçôes sobre a pesquisa e assinaram o Termo de Consentimento Livre e Esclarecido (TCLE).

O presente trabalho foi composto por pacientes portadores de câncer hematológico em tratamento, avaliados durante o primeiro, quinto e o décimo dia de internamento hospitalar. Cinquenta e seis pacientes portadores de leucemia e linfoma, maiores de 18 anos, de ambos os sexos, admitidos na enfermaria onco-hematológica, foram considerados elegíveis para participar do estudo; entretanto, 31 pacientes foram excluídos porque não aceitaram participar do estudo, pacientes que estavam em isolamento aéreo ou de contato, instabilidade clínica, dificuldade de compreensão, e com comorbidades neuromusculoesqueléticas, dez pacientes foram excluídos porque receberam alta antes do décimo dia de internamento hospitalar.

A coleta de dados foi realizada em três períodos durante o internamento hospitalar, com preenchimento de ficha de avaliação com informações sociodemográficas (idade, sexo, estado civil, ocupação), medidas antropométricas de peso e altura e índice de massa corpórea (IMC) coletados no prontuário eletrônico. Além disso, cada paciente foi avaliado com instrumentos que mensuram a pressão inspiratória máxima (PIM) e a pressão expiratória máxima (PEM), e o nível de capacidade funcional por pontuação obtida na KPS. Os pacientes foram avaliados por um único avaliador, durante 40 minutos.

A musculatura respiratória foi avaliada pelo manovacuômetro analógico (WIKA, modelo 611.10, Brasil) cuja escala apresenta intervalos de $10 \mathrm{cmH}_{2} \mathrm{O}$ e variação de $-150 \mathrm{a}+150 \mathrm{cmH}_{2} \mathrm{O}$, utilizado com a finalidade de quantificar a força dos músculos da 
mecânica respiratória. A PIM e a PEM são verificadas a partir do volume residual e a capacidade pulmonar total, respectivamente, por meio de esforços voluntários. Os pacientes foram, a todo tempo, incentivados pelo avaliador a desempenhar esforços máximos, sendo realizadas três manobras em cada avaliaçáo, e anotada a de maior valor. $\mathrm{O}$ esforço inspiratório máximo e o expiratório máximo foram mantidos por três segundos. O equipamento foi utilizado seguindo a técnica de mensuração padronizada pela American Thoracic Society (ATS) e European Respiratory Society (ERS) ${ }^{12}$. O bocal adaptado ao manovacuômetro possuía um orifício de $2 \mathrm{~mm}$, para evitar contração dos músculos bucinadores em razão da pressão intraoral ${ }^{13}$.

Para determinação do nível funcional e a evoluçâo do paciente com o tratamento, foi preenchida a escala KPS, que consiste em 11 pontos variando de 0 a 100 , onde, quanto menor a pontuação, pior a expectativa de recuperação da enfermidade ou retorno às atividades normais. Ela descreve o perfil da população com câncer, classificando os pacientes de acordo com o grau de suas aptidóes e deficiências funcionais em três grupos, conforme o seu grau de aptidáo física para trabalhar e exercer tarefas cotidianas e necessidades de cuidados: capaz de realizar atividade normal, não precisa de cuidado especial (80\% a $100 \%)$; incapaz de trabalhar, capaz de viver em casa e cuidar da maioria das necessidades pessoais, mas é necessária uma quantidade variável de assistência (50\% a $70 \%)$; incapaz de cuidar de si mesmo, requer o equivalente de cuidado institucional ou hospitalar, a doença pode estar progredindo rapidamente ( $0 \%$ a $40 \%)$, além de comparar a eficiência da utilização de diferentes terapias, o que possibilita determinar o prognóstico individual para cada paciente $\mathrm{e}^{14,15}$.

A análise dos dados demográficos e clínicos foi realizada por intermédio de estatísticas descritivas. As variáveis contínuas são expressas em medidas de tendência central e dispersão, e as de variáveis dicotômicas ou categóricas com medidas de frequência. Para avaliar a normalidade dos dados, foi realizado o teste de Kolmogorov-Smirnov e, para comparação das variáveis com o tempo de internamento, o teste Kruskal-Wallis. O nível de significância adotado foi $\mathrm{p}<0,05$. A análise estatística foi realizada utilizando o software Statistical Package for Social Sciences, versão 14.0 (SPSS Inc Chicago, IL, EUA).

\section{RESULTADOS}

Foram avaliados 15 pacientes no período de fevereiro a julho de 2017. Entre eles, oito pacientes com leucemia (53\%) e sete com linfoma (47\%). Os dados demográficos e clínicos estão descritos na Tabela 1.
Tabela 1 Dados demográficos e clínicos dos pacientes onco-hematológicos

\begin{tabular}{l|c}
\hline \multicolumn{1}{c|}{ Variável } & $\mathbf{n = 1 5}$ \\
\hline Idade: mediana (intervalo) - anos & \\
Masculino & $37(20-66)$ \\
Feminino & $29(20-54)$ \\
\hline Hemoglobina (g/dl) & $8,1(6,8-11)$ \\
\hline Altura (cm) & $1,63 \pm 0,23$ \\
\hline Índice de massa corpórea & $21,7 \pm 0,36$ \\
\hline Sexo & $\mathrm{n}(\%)$ \\
Masculino & $7(47 \%)$ \\
Feminino & $8(53 \%)$ \\
\hline Tipo de câncer & $\mathrm{n} \mathrm{( \% )}$ \\
Leucemia & $8(53 \%)$ \\
Linfoma & $7(47 \%)$ \\
\hline Quimioterapia & $\mathrm{n}(\%)$ \\
Ciclo 1 & $11(73,3 \%)$ \\
Ciclo 3 & $2(13,3 \%)$ \\
Ciclo 4 & $2(13,3 \%)$ \\
\hline Trabalha & $\mathrm{n} \mathrm{( \% )}$ \\
Sim & $4(27 \%)$ \\
Não & $11(73 \%)$ \\
\hline Estado civil & $\mathrm{n}(\%)$ \\
Com parceiro & $9(60 \%)$ \\
Sem parceiro & $6(40 \%)$ \\
\hline
\end{tabular}

Nota: Resultados expressos em mediana (mínimo-máximo); porcentagem (\%) ou em média \pm desvio-padrăo.

A PIM não apresentou variação significativa durante o primeiro, o quinto e o décimo dia de internamento hospitalar, sendo observada a mediana $60 \mathrm{cmH}_{2} \mathrm{O}$. Em todas as avaliaçóes, a PIM mínima foi $30 \mathrm{cmH}_{2} \mathrm{O}$; o primeiro quartil (Q1-25\%) mostra que os pacientes apresentaram PIM de $50 \mathrm{cmH}_{2} \mathrm{O}$; e, no terceiro quartil (Q3-75\%), do primeiro e décimo dias, correspondeu a 80 $\mathrm{cmH}_{2} \mathrm{O}$. Alguns pacientes apresentaram valores de PIM mais altos do que a mediana do grupo (Figura 1).

$\mathrm{Na}$ avaliação da PEM, observou-se variação entre a mediana durante o internamento hospitalar. A PEM foi maior no quinto dia, $60 \mathrm{cmH}_{2} \mathrm{O}$. Verificou-se redução significativa da PEM entre o quinto e o décimo dia de internamento hospitalar ( $\mathrm{p}=0,000)$, uma vez que, no quinto dia de permanência dos pacientes no hospital, houve um aumento da PEM. Em todas as avaliaçóes, alguns pacientes apresentaram PEM maiores do que a do grupo (Figura 2).

A mediana de funcionalidade dos pacientes durante o primeiro, o quinto e o décimo dia de internamento hospitalar não teve variação, significando $70 \%$, porém observou-se que, no décimo dia, a amplitude máxima de funcionalidade correspondeu a Q3-75\%, diferente do primeiro dia, porém não foi estatisticamente significativo, $\mathrm{p}=1,121$, Figura 3 . 


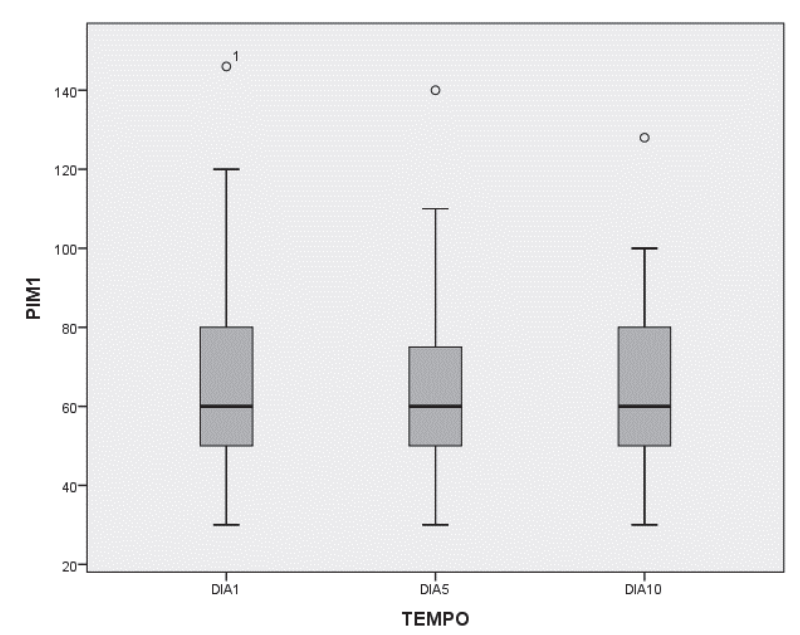

Figura 1. Efeitos da hospitalização na PIM dos pacientes onco-hematológicos

Nota: Os pacientes foram avaliados no HUPES no primeiro, quinto e décimo dia de internamento $(\mathrm{n}=15)$.

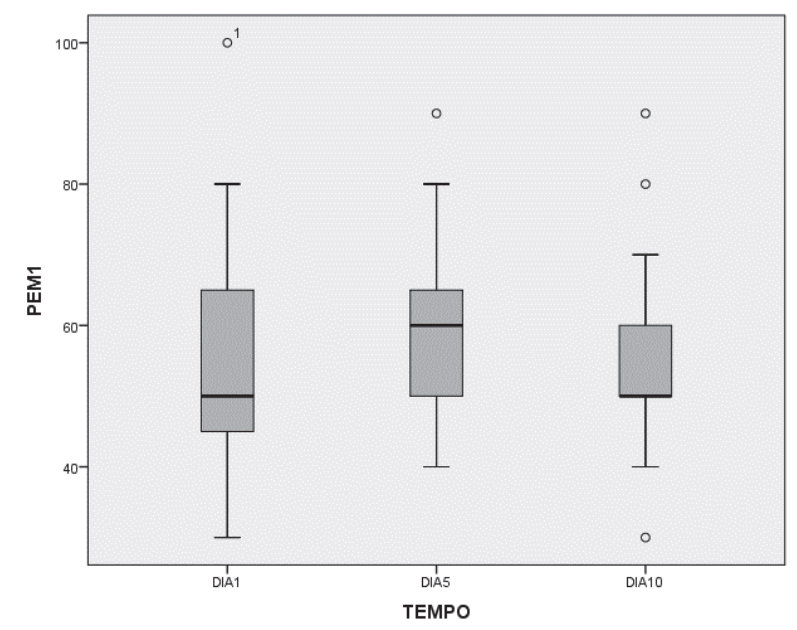

Figura 2. Ełeitos da hospitalização na PEM dos pacientes onco-hematológicos

Nota: Os pacientes foram avaliados no HUPES no primeiro, quinto e décimo dia de internamento $(\mathrm{n}=15)$.

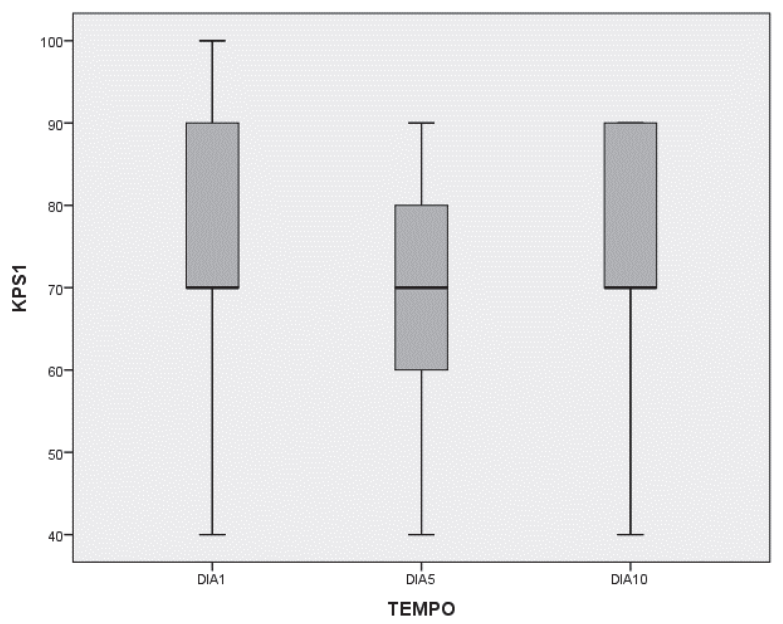

Figura 3. Ełeitos da hospitalização sobre o nível funcional dos pacientes onco-hematológicos

Nota: Os pacientes foram avaliados no HUPES no primeiro, quinto e décimo dia de internamento $(\mathrm{n}=15)$.
A avaliação da força muscular respiratória e o nível funcional dos pacientes, de acordo o sexo, encontram-se na Tabela 2.

Tabela 2. Avaliação da força muscular respiratória e funcionalidade dos pacientes onco-hematológicos de acordo com o sexo

\begin{tabular}{l|c|c|c}
\hline \multicolumn{1}{c|}{ Variáveis } & $\mathbf{1}^{\circ}$ dia & $5^{\circ}$ dia & $\mathbf{1 0}^{\circ}$ dia \\
\hline $\begin{array}{l}\text { PIM F } \\
\left(\mathrm{cmH}_{2} \mathrm{O}\right)\end{array}$ & $60(30-80)$ & $60(40-80)$ & $60(30-100)$ \\
\hline $\begin{array}{l}\mathrm{PIM} \mathrm{M} \\
\left(\mathrm{cmH}_{2} \mathrm{O}\right)\end{array}$ & $70(40-146)$ & $50(30-140)$ & $60(50-128)$ \\
\hline $\begin{array}{l}\mathrm{PEM} \mathrm{F} \\
\left(\mathrm{cmH}_{2} \mathrm{O}\right)\end{array}$ & $55(30-80)$ & $50(40-80)$ & $55(30-80)$ \\
\hline $\begin{array}{l}\mathrm{PEM} \mathrm{M} \\
\left(\mathrm{cmH}_{2} \mathrm{O}\right)\end{array}$ & $50(30-100)$ & $60(40-90)$ & $50(40-90)$ \\
\hline KPS M (\%) & $90(40-90)$ & $80(40-90)$ & $80(40-90)$ \\
\hline KPS F (\%) & $70(50-70)$ & $60(50-90)$ & $70(50-90)$ \\
\hline
\end{tabular}

Legendas: PIM $\mathrm{F}=$ pressão inspiratória máxima feminina; PIM $\mathrm{M}=$ pressão inspiratória máxima masculina; $\mathrm{PEM} \mathrm{F}$ = pressão expiratória máxima feminina; PEM M = pressão expiratória máxima masculina; KPS M = escala de desempenho funcional - grupo masculino; KPS F = escala de desempenho funcional - grupo feminino.

Nota: Resultados expressos em mediana (mínimo-máximo).

\section{DISCUSSÃO}

Pouco foi publicado sobre a funcionalidade de pacientes onco-hematológicos no Brasil. O presente estudo analisa os aspectos relacionados às repercussóes do tempo de internamento sobre a funçáo respiratória e o nível funcional dos pacientes oncológicos, visto que elas ainda não são bem conhecidas.

Com isso, torna-se imprescindível a avaliação da força muscular respiratória e da capacidade funcional para auxiliar na compreensão de fatores que vão além do processo de adoecimento, abrangendo outros domínios, principalmente os relacionados às $\mathrm{ABVD}$ e às AIVD, permitindo uma abrangência maior no tratamento. Conhecer o perfil dos pacientes hospitalizados permite elaborar estratégias preventivas de comorbidades associadas ao tratamento com intuito de reduzir as limitaçóes funcionais?.

O presente estudo mostra que os homens são mais velhos que as mulheres, o IMC estava dentro da faixa de normalidade, e a maioria dos pacientes avaliados encontrava-se no primeiro ciclo de quimioterapia - tratamento que envolve etapas e tem seus efeitos dependentes do tipo de fármaco administrado ${ }^{16}$. Todos os pacientes tinham graus variados de anemia, sendo associados à alteração da hematopoese ${ }^{17}$. Carbonell et al. ${ }^{18}$ demonstraram em estudo com 82 pacientes que o desempenho funcional baixo na KPS é influenciado por níveis reduzidos de hemoglobina.

Não houve variabilidade significativa na PIM ao longo dos dias de internamento hospitalar, isso pode 
ser associado ao perfil dos pacientes admitidos nessa enfermaria, pois a maioria não estava restrita ao leito. Porém, alguns pacientes apresentaram uma PIM muito baixa. Corroborando os dados deste estudo, Grande et al. ${ }^{19}$, em sua pesquisa com 93 pacientes internados, sendo a maior parte com neoplasia, também não encontraram redução da PIM dos pacientes que realizavam fisioterapia $(55,8 \pm 28,5$ a $53,3 \pm 28,5)(\mathrm{p}=0,087)$, diferentemente de Suesada et al. ${ }^{20}$, que, em seu estudo, avaliaram 78 pacientes antes e depois da hospitalização de cinco dias, e encontraram redução da PIM $(83,3 \pm 25,0$ para $70,9 \pm 23,1)$ $\mathrm{cmH}_{2} \mathrm{O}(\mathrm{p}=<0,001)$. Valores de PIM com um terço do normal são preditores de falência respiratória e hipercarbia $\left(\mathrm{PaCO}_{2}>45 \mathrm{mmHg}\right)^{12}$.

Quanto à PEM, houve uma alteração dos valores ao longo da permanência hospitalar, sendo observado declínio, ao comparar o quinto e o décimo dia $(\mathrm{p}=0,000)$. Essa redução pode ser relacionada tanto pela hospitalização quanto pelos efeitos da quimioterapia, pois, no décimo dia, os pacientes encontram-se no "nadir", período entre a administração dos medicamentos citotóxicos e a ocorrência da menor contagem hematológica, podendo apresentar quadro de dor, fadiga, além de estarem mais propensos a permanecer acamados ${ }^{21}$. Grande et al. ${ }^{19}$ observaram relação entre o tempo de internaçáo e os valores de PEM $(53,2 \pm 25,1$ para $50,7 \pm 26,1)(p=0,054)$, confirmando nossos achados. Suesada et al. ${ }^{20}$ também encontraram redução de PEM de $98,3 \pm 21,9$ para $89,3 \pm 24,0 \mathrm{cmH}_{2} \mathrm{O}$. Uma PEM abaixo de $60 \mathrm{cmH}_{2} \mathrm{O}$ é preditora de tosse ineficaz, com tendência à retenção de secreção ${ }^{12}$.

Em relação ao sexo, não houve diferença entre as medianas de PIM e PEM entre os grupos masculino e o feminino. Entretanto, os homens não mantiveram a pressão respiratória durante o internamento, enquanto as mulheres conseguiram manter ao longo do quinto e décimo dia. Durante a hospitalização, a redução do condicionamento físico é associada ao processo de adoecimento, gerando prejuízos que interferem diretamente na funcionalidade $\mathrm{e}$ qualidade de vida dos doentes ${ }^{22}$.

A maioria dos pacientes da nossa pesquisa tinha bom nível funcional no primeiro dia de internamento, sendo aptos a realizar ABVD sem ajuda e a trabalhar, pontuação obtida entre $80 \%$ a $100 \%$, porém houve redução da funcionalidade no quinto dia; isso pode ser justificado pela incorporaçáo do acesso venoso periférico, o peso da bomba de quimioterapia, que limita algumas atividades, sendo responsável por necessidade de auxílio, nível de 50\% a 70\%. No décimo dia, a maioria dos pacientes possuía um bom nível funcional. Entretanto, o máximo de funcionalidade alcançada foi menor do que na primeira avaliação.

Essa evidência pode ser justificada, em parte, pelo nível da assistência à saúde fornecido aos pacientes durante o internamento, visto que a maioria deles dispóe de mais de um atendimento fisioterapêutico por dia, ficando evidenciada a importância do fisioterapeuta como profissional de saúde no decorrer do tratamento dos pacientes; além disso, associou-se essa melhora à adaptabilidade do paciente no ambiente hospitalar, principalmente no que tange à esfera da relação emocional, pela percepçáo de afeto entre o terapeuta e o paciente, com enfoque no apoio psicossocial fornecido tanto pela equipe multiprofissional quanto pela família no auxílio e estímulo ao tratamento .

Os achados deste estudo corroboram, também, os encontrados por $\mathrm{Da}$ Costa et al..$^{23}$, que constataram que a internaçáo superior a sete dias não afeta a independência funcional dos pacientes. De acordo com Wedding et al. ${ }^{24}$, a funcionalidade é afetada com o envelhecimento; a mediana da KPS no seu trabalho foi de $80 \%$ em pacientes jovens.

No presente estudo, foi comprovado que, mesmo com a neoplasia, os pacientes onco-hematológicos apresentam um bom nível funcional, porém, sendo modificado durante o internamento, isso pode ser associado ao ciclo de quimioterapia, uma vez que a maioria estava no primeiro ciclo, não tendo efeitos táo agressivos ao organismo.

De acordo a mediana, os homens foram mais funcionais do que as mulheres em todas as avaliações. Embora existam comorbidades semelhantes, eles demonstraram mais aptidão para realizar ABVD e AIVD. Contudo, tiveram um declínio da funcionalidade durante o quinto e décimo dia, sendo evidenciados os efeitos do tratamento na função física dos pacientes internados ${ }^{21}$. Apesar de as mulheres apresentarem um nível funcional menor do que os homens, elas conseguiram manter sua funcionalidade durante a hospitalização. Esses dados sugerem que pacientes que mantêm um bom nível de força muscular respiratória têm tendência a terem uma boa capacidade para realizar atividades diárias mais complexas e que envolvem maior gasto energético.

Todavia, o presente estudo apresentou limitaçóes importantes quanto ao número reduzido da amostra, às perdas de pacientes e às dificuldades de avaliaçáo, em virtude de alguns procedimentos no curso do tratamento e o ciclo de quimioterapia que pode ter interferido nos resultados. Além disso, a escassez de estudos com o mesmo objetivo limitou a comparação dos nossos dados.

\section{CONCLUSÃO}

O internamento hospitalar pode alterar a força muscular respiratória e a capacidade funcional de pacientes hospitalizados. Tendo em vista os aspectos observados, o tempo de internamento altera a PEM dos pacientes onco-hematológicos, mas não reduz a PIM e a funcionalidade. 
Entretanto, mais estudos são necessários para comprovar os efeitos do internamento sobre a musculatura respiratória e a funcionalidade dos pacientes.

\section{CONTRIBUIÇÕES}

Natali dos Santos Nascimento participou da concepção do projeto, delineamento, busca coleta, interpretaçáo dos dados, redação e revisão do artigo científico. Nicolly Del Carmen Parra Molina Mattos, Samara de Souza Marques e Thais Conceição Cruz participaram da concepção do projeto, delineamento, busca, coleta, interpretação dos dados e revisão do artigo. Camila Reinbold Rezende participou da concepção, delineamento do projeto e revisão do artigo. Cássio Magalhães da Silva e Silva participou do delineamento, análise estatística e revisão do artigo.

\section{DECLARAÇÃO DE CONFLITO DE INTERESSES}

Nada a declarar.

\section{FONTES DE FINANCIAMENTO}

Não há.

\section{AGRADECIMENTOS}

Os autores agradecem aos profissionais da enfermaria onco-hematológica do Hupes.

\section{REFERÊNCIAS}

1. Calefi KAC, Rocha V, Nabhan SK, Maftum MA, Kalinke LP, Mantovani MF. Qualidade de vida do paciente com neoplasia hematológica submetido à quimioterapia. Rev Min Enferm. 2014;18(1):41-47. doi: http://www.dx.doi. org/10.5935/1415-2762.20140004.

2. Instituto Nacional de Câncer José Alencar Gomes da Silva. Estimativa 2018: incidência de câncer no Brasil [Internet]. Rio de Janeiro: INCA; 2017. [acesso 2018 Out 15]. Disponível em: http://www1.inca.gov.br/inca/ Arquivos/estimativa-2018.pdf.

3. Battaglini CL, Bottaro M, Campbell JS, Novaes J, Simáo R. Atividade física e níveis de fadiga em pacientes portadores de câncer. Rev Bras Med Esporte. 2004;10(2):98-104. doi: http://dx.doi.org/10.1590/ S1517-86922004000200004.

4. Macedo TMF, Oliveira KMC, Melo JBC, Medeiros MG, Medeiros Filho WC, Ferreira GMH, et al. Treinamento muscular inspiratório em crianças com leucemia aguda: resultados preliminares. Rev Paul Pediatr. 2010;28(4):352-8. doi: http://dx.doi.org/10.1590/ S0103-05822010000400011.
5. Ferreira EVM. Respiratory muscles: myths and secrets. J Bras Pneumol. 2015;41(2): 107-9. doi: http://dx.doi. org/10.1590/S1806-37132015000200002.

6. Reis JS, Dantas MS, Silva CB, Valverde L, Landeiro, RBR. Caracterização da Força Muscular Respiratória e da Capacidade Funcional de Pacientes Internados em uma Enfermaria. Rev Fisioter Saude Funcional [Internet]. 2012 [acesso 2017 Maio 03];1(2):3-9. Disponível em: http://www.fisioterapiaesaudefuncional.ufc.br/index. php/fisioterapia/article/view/83/pdf_2

7. Syabbalo N. Assessment of respiratory muscle function and strength. Postgrad Med J [Internet]. 1998 Apr. [cited 2017 Jun 15]; 74(870):208-215. Disponível em: https:// www.ncbi.nlm.nih.gov/pmc/articles/PMC2360860/.

8. Chang VT, Hwang SS, Feuerman M, Kasimis BS, Thaler HT. The memorial symptom assessment scale short form (MSAS-SF): validity and reliability. Cancer. 2000;89(5):1162-71. doi: https://doi. org/10.1002/1097-0142(20000901)89:5<1162::AIDCNCR26>3.0.CO;2-Y.

9. Pereira EEB, Santos NB, Sarges ESNF. Avaliação da capacidade funcional do paciente oncogeriátrico hospitalizado. Rev Pan-Amaz Saude. 2014;5(4):37-44. doi: http://dx.doi.org/10.5123/S2176-62232014000400005.

10. César CC, Mambrini JVM, Ferreira FR, Lima-Costa MF. Capacidade funcional de idosos: análise das questôes de mobilidade, atividades básicas e instrumentais da vida diária via Teoria de resposta ao Item. Cad Saúde Pública. 2015;31(5):931-945. doi: http://dx.doi. org/10.1590/0102-311X00093214.

11. Silva BCA, Amorim D, Salício VAMM, Salício MA, Shimoya-Bittencourt W. Avaliação da funcionalidade respiratória em pacientes com tempo prolongado de internação hospitalar. J Health Sci Inst [Internet]. 2014 [acesso 2017 Jun. 13];32(4): 433-8. Disponível em: https://www.unip.br/comunicacao/publicacoes/ics/ edicoes/2014/04_out-dez/V32_n4_2014_p433a438.pdf

12. Bessa EJC, Lopes AJ, Rufino R. A importância da medida da força muscular respiratória na prática da pneumologia. Pulmáo RJ [Internet]. 2015 [acesso 2017 Jun 30];24(1):37-41. Disponível em: http://www.sopterj. com.br/wp-content/themes/_sopterj_redesign_2017/_ revista/2015/n_01/10.pdf.

13. Costa D, Gonçalves HA, Lima LP, Ike D, Cancelliero KM, Montebelo MIL. Novos valores de referência para pressōes respiratórias máximas na população brasileira. J Bras Pneumol. 2010;36(3):306-312. doi: http://dx.doi. org/10.1590/S1806-37132010000300007.

14. Karnofsky DA, Abelmann WH, Craver LF, Burchenal JH. The use of the nitrogen mustards in the palliative treatment of carcinoma: with particular reference to bronchogenic carcinoma. Cancer. 1948;1(4):634-656. doi: https:// doi.org/10.1002/1097-0142(194811)1:4<634::AIDCNCR2820010410>3.0.CO;2-L. 
15. Yates JW, Chalmer B, Mckegney FP. Evaluation of patients with advanced cancer using the karnofsky performance status. Cancer. 1980;45(8):2220-2224. doi: https://doi. org/10.1002/1097-0142(19800415)45:8<2220::AIDCNCR2820450835>3.0.CO;2-Q.

16. American Cancer Society. Typical Treatment of Acute Myeloid Leukemia (Except APL). [Internet]. [place unknown]: American Cancer Society; 2017 [cited 2017 July 07]. Available from https://www.cancer.org/cancer/acute-myeloidleukemia/treating/typical-treatment-of-aml.html

17. Rose-Inman H, Kuehl D. Acute Leukemia. Emerg Med Clin North Am. 2014;32(3): 579-96. doi: https://doi. org/10.1016/j.emc.2014.04.004.

18. Carbonell ALI, Salhab RM, Giampaoli V, Cendoroglo MS, Chauffaille ML. A model for the functional assessment of elderly with myeloid neoplasms. Rev Bras Hematol Hemoter. 2015;37(2):109-14. doi: http:// dx.doi.org/10.1016/j.bjhh.2015.02.003.

19. Grande GHD, Napoleão LL, Lima FF, Braun C, Barbosa TC, Padulla SAT. Influência da fisioterapia na força muscular respiratória e força de preensão palmar de pacientes hospitalizados. Colloq Vitae. 2015;7(1):69-75. doi: http://dx.doi.org/10.5747/cv.2015.v07.n1.v127.

20. Suesada MM, Martins MA, Carvalho CRF. Effect of Short-Term Hospitalization on Functional Capacity in Patients Not Restricted to Bed. Am J Phys Med Rehabil. 2007;86(1): 455-62. doi: http://dx.doi.org/10.1097/ PHM.0b013e31805b7566.

21. Ávila FF, Soares MBO, Silva SR. Perfil hematológico e bioquímico sérico de pacientes submetidas à quimioterapia antineoplásica. REAS [Internet]. 2013 [acesso 2017 Ago 01];2(2):32-45. Disponível em: http://seer.uftm.edu.br/ revistaeletronica/index.php/enfer/article/view/385/406.

22. Ehlenbach WJ, Larson EB, Curtis JR, Hough CL. Physical function and disability after acute care and critical illness hospitalizations in a prospective cohort of older adults. J Am Geriatr Soc. 2015;63(10):2061-2069. doi: https://doi.org/10.1111/jgs.13663.

23. Da Costa FM, Correa ADB, Narala Neto E, Vieira EMM, Nasrala MLS, Lima E, et al. Avaliação da Funcionalidade Motora em Pacientes com Tempo Prolongado de Internação Hospitalar. UNOPAR Cient Ciênc Biol Saúde. [Internet]. 2014 [acesso 2017 Jul 31];16(2):8791. Disponível em: http://www.pgsskroton.com.br/seer/ index.php/JHealthSci/article/viewFile/486/456

24. Wedding U, Röhrig B, Klippstein A, Fricke HJ, Sayer HG, Höffken K. Impairment in functional status and survival in patients with acute myeloid leukaemia. J Cancer Res Clin Oncol. 2006;132(10):665-71. doi: https://doi.org/10.1007/s00432-006-0115-7. 